\title{
Comparison of Iterative Model Reconstruction versus Filtered Back-Projection in Pediatric Emergency Head CT: Dose, Image Quality, and Image-Reconstruction Times
}

\author{
(D) R.N. Southard, DD.M.E. Bardo, (D) M.H. Temkit, DM.A. Thorkelson, DR.A. Augustyn, and (D) C.A. Martinot
}

\begin{abstract}
BACKGROUND AND PURPOSE: Noncontrast CT of the head is the initial imaging test for traumatic brain injury, stroke, or suspected nonaccidental trauma. Low-dose head CT protocols using filtered back-projection are susceptible to increased noise and decreased image quality. Iterative reconstruction noise suppression allows the use of lower-dose techniques with maintained image quality. We review our experience with children undergoing emergency head CT examinations reconstructed using knowledge-based iterative model reconstruction versus standard filtered back-projection, comparing reconstruction times, radiation dose, and objective and subjective image quality.
\end{abstract}

MATERIALS AND METHODS: This was a retrospective study comparing 173 children scanned using standard age-based noncontrast head CT protocols reconstructed with filtered back-projection with 190 children scanned using low-dose protocols reconstructed with iterative model reconstruction. ROls placed on the frontal white matter and thalamus yielded signal-to-noise and contrast-to-noise ratios. Volume $\mathrm{CT}$ dose index and study reconstruction times were recorded. Random subgroups of patients were selected for subjective image-quality review.

RESULTS: The volume CT dose index was significantly reduced in studies reconstructed with iterative model reconstruction compared with filtered back-projection, (mean, $24.4 \pm 3.1 \mathrm{mGy}$ versus $31.1 \pm 6.0 \mathrm{mGy}, P<.001$ ), while the SNR and contrast-to-noise ratios improved 2 -fold $(P<.001)$. Radiologists graded iterative model reconstruction images as superior to filtered back-projection images for gray-white matter differentiation and anatomic detail $(P<.001)$. The average reconstruction time of the filtered back-projection studies was 101 seconds, and with iterative model reconstruction, it was 147 seconds $(P<.001)$, without a practical effect on work flow.

CONCLUSIONS: In children referred for emergency noncontrast head CT, optimized low-dose protocols with iterative model reconstruction allowed us to significantly reduce the relative dose, on average, $22 \%$ compared with filtered back-projection, with significantly improved objective and subjective image quality.

ABBREVIATIONS: ASIR = adaptive statistical iterative reconstruction; $C N R=$ contrast-to-noise ratio; CTDI $_{\text {vol }}=$ volume $C T$ dose index; FBP $=$ filtered backprojection; $I M R=$ iterative model reconstruction; $M B I R=$ model-based iterative reconstruction; $\mathbb{I R}=$ iterative reconstruction

C hildren younger than 18 years of age constitute approximately one-fifth of the 130 million annual visits to hospitalaffiliated emergency departments in the United States. ${ }^{1}$ Trauma is a leading cause of morbidity and mortality in children older than

Received October 15, 2018; accepted after revision February 27, 2019. From the Departments of Medical Imaging (R.N.S., D.M.E.B., M.A.T., R.A.A., C.A.M.) and Clinical Research (M.H.T.), Phoenix Children's Hospital, Phoenix Arizona.

Paper previously presented at: Society for Pediatric Radiology Annual Meeting and Postgraduate Course, May 15-19, 2018; Nashville, Tennessee.

Abstract reuse permission from Springer Nature/RightLink license No. 4366020744406, obtained June 11, 2018.

Please address correspondence to Richard N. Southard, MD, Vice Chair of Clinical Operations, Director of CT and Cardiac Imaging, Department of Medical Imaging, Phoenix Children's Hospital, 1919 East Thomas Rd, Main Tower/Radiology, Phoenix, AZ 85016; e-mail: rsouthard@phoenixchildrens.com

http://dx.doi.org/10.3174/ajnr.A6034
1 year, with head trauma representing nearly $80 \%$ of these injuries. Noncontrast head CT is often the first-line study for evaluation of traumatic brain injury or suspected nonaccidental trauma in children. ${ }^{2}$ Various dose-reduction strategies are used by vendors and radiologists to reduce CT radiation exposure in children. ${ }^{3,4}$ Minimizing radiation exposure while maintaining image quality of brain CT is challenging because low-contrast lesion detectability, detection of subtle bleeds, and anatomic detail, especially in the posterior fossa, may be affected by noise, artifacts, and reduced contrast-to-noise ratio (CNR) that accompanies lower-dose techniques. ${ }^{5}$ The use of lower-dose techniques by lowering the tube voltage or current, with filtered back image reconstruction, is susceptible to increased image noise, decreased spatial resolution, and low-contrast lesion detectability and overall reduced reader confidence. 
Image reconstruction with iterative reconstruction (IR) uncouples noise reduction and spatial resolution and allows the use of lower-dose techniques with maintained image quality. ${ }^{6,7}$ There are different types of iterative reconstructions: those that denoise image domain space alone; those that denoise in both projection and image space; and pure, systems- or model-based iterative reconstruction (MBIR), which takes into consideration all aspects of the imaging chain including system optics and, by virtue of complex computational algorithms used, has longer reconstruction times, which may prevent its use in the emergency setting. Overuse of noise-suppression strength may also result in an undesirable overly smooth image texture with loss of anatomic edge detail presumably due to a shift in spatial frequency distribution of image noise. We review our experience with children undergoing emergency head CT examinations reconstructed using knowledge-based iterative model reconstruction (IMR; Philips Healthcare, Best, the Netherlands) versus standard filtered backprojection (FBP) reconstruction, comparing reconstruction times, radiation dose, and objective and subjective image quality.

\section{MATERIALS AND METHODS \\ Design}

This institutional review board-approved and Health Insurance Portability and Accountability Act-compliant retrospective review compared 400 nonrandomized consecutive patients referred from the emergency department for urgent head CT examinations between January and March 2016 performed using either standard age-based protocols and FBP image reconstruction or reduced-dose age-based protocols and IMR. The institutional review board waived the need for patient consent.

\section{Patient Groups}

All children referred from the emergency department for urgent head CT without contrast $+3 \mathrm{D}$ reconstruction (Current Procedural Terminology: 70450/76376) in 2016 were identified in our image data base. The first 200 consecutive patients imaged on the 64-slice multidetector row CT scanner situated in the emergency department with FBP image reconstruction, and the first 200 consecutive patients imaged on the 256-slice multidetector row CT scanner in the main department with image reconstruction using IMR were eligible. Excluded were patients with repeat studies, marked motion, and multiple implanted metallic leads or when the incorrect age-based CT protocol was used. Patient age (months) and sex were recorded for each subject.

\section{Data Acquisition and Reconstruction}

We used 4 age-based protocols for unenhanced CT head examinations: younger than 1.5 years, $1.5-6$ years, $7-12$ years, and older than 13 years. Image-acquisition parameters of the standard-dose studies performed on the emergency department scanner (Brilliance 64-Slice; Philips Healthcare) included a collimation of $64 \times$ $0.625 \mathrm{~mm}$, pitch of 0.64 , rotation time of 0.5 seconds, tube voltage of $120 \mathrm{kV}$, and tube currents corresponding to the 4 age-based protocols of 200, 225, 300, or 350 mAs. Scan parameters for the low-dose studies performed on the main department scanner (Brilliance iCT 256-Slice; Philips Healthcare) included collimation of $64 \times 0.625 \mathrm{~mm}$, with a pitch of 0.39 , rotation time of 0.4 seconds, and age-dependent tube currents of 160, 168, 200, or 210 $\mathrm{mAs}$. The FBP standard-dose examinations were reconstructed with a UB (soft) convolution kernel, section-thickness of $3 \mathrm{~mm}$ with 3-mm increments, and high resolution, and the IR low-dose examinations were reconstructed with a mild noise-reduction $\mathrm{IMR}_{\text {BRAIN } 1}$ routine convolution kernel, a section-thickness of $3 \mathrm{~mm}$ with 3-mm increments, and high resolution.

Both scanners were surveyed and tested by a certified medical CT physicist in accordance with the American College of Radiology CT Accreditation Program as part of our routine departmental quality-assurance program. Comparison of the calculated clinical examination dose estimate and the measured volume CT dose index $\left(\mathrm{CTDI}_{\mathrm{vol}}\right)$ reported by the scanner yielded a percentage difference of $11.86 \%$ for pediatric head ( 1 year of age) and $2.48 \%$ for adult head CT protocols on the iCT 256-Slice multidetector row CT scanner, and $0.80 \%$ and $2.60 \%$ on the Brilliance 64 -Slice multidetector row CT scanner.

\section{Radiation Dose Measurement}

The estimated radiation dose $\mathrm{CTDI}_{\mathrm{vol}}$ was recorded for each study.

\section{Reconstruction Time}

Reconstruction times were determined by recording the millisecond image-creation times for the first image and the last image reconstructed for each examination, as obtained from the information tile available for each individual image on the PACS workstation.

\section{Quantitative Analysis}

All images were viewed on a dedicated PACS on a calibrated dedicated diagnostic workstation in our department. For each study, a single representative image at the level of the lateral ventricles was selected to include both the thalamus and frontal white matter, and 1 radiologist with 25 years' experience who was not involved in the blinded subjective image-quality review placed equal ROIs measuring between 6 and $9 \mathrm{~mm}$ on the right thalamus deep gray matter and right frontal white matter to maintain uniformity. Measurements of mean CT density (Hounsfield unit) and SD yielded the signal-tonoise ratio for the white matter and thalamus $\left(\mathrm{SNR}_{\text {White Matter }}\right.$ and $\mathrm{SNR}_{\text {Thalamus }}$ ) using the formula SNR $=\mathrm{HU} / \mathrm{SD}$, and $\mathrm{CNR}$ using the formula $\mathrm{CNR}=\left(\mathrm{HU}_{\text {Thalamus }}-\mathrm{HU}_{\text {White Matter }}\right) /\left[\left(\mathrm{SD}_{\text {Thalamus }}+\right.\right.$ $\left.\left.\mathrm{SD}_{\text {White Matter }}\right) / 2\right]$.

\section{Qualitative Analysis}

Subjective image quality was assessed by selecting a randomized de-identified age-group-matched subgroup from both the FBP and IR groups using a random number generator (http://www. randomnumbergenerator.com/) ( $n=40$; power index, $>80 \%)$. Qualitative analysis of the 3-mm images was independently performed by 2 board-certified, subspecialty-certified neuroradiology attending radiologists with 16 and 17 years' experience, and scores were reported assessing gray-white matter differentiation and anatomic detail using a 5-point scale of 1, poor; 2, fair; 3, average; 4, good; and 5, excellent. Reviewers were blinded to the scanner, scan parameters, reconstruction settings, and slice thickness.

AJNR Am J Neuroradiol 40:866-71 May 2019 www.ajnr.org 


\section{Statistical Analysis}

Group comparisons were conducted using 2 independentsamples equal variances $t$ tests for continuous variables and the $\chi^{2}$ test for categoric variables. The Pearson correlation coefficient and the resulting $P$ value for statistical significance were determined, with the significance level at $P<.05$. Subjective image-quality scores were combined as an average for each patient, and the intraclass correlation coefficient and correlation coefficient for the individual physician grades were calculated. Power and sample-size calculations for subjective imagequality scoring using a 2-group independent-samples $t$ test were used to determine the required number of subjects needed to detect a mean difference of 1.0 between FBP and IR. The power calculations were conducted using the procedure Proc Power available in the SAS statistical software. Statistical analyses were performed using SAS 9.4 (SAS Institute, Cary North Carolina) and R Studio, Version 1.0.153 (http://rstudio.org/ download/desktop).

\section{RESULTS}

\section{Patient Groups}

A total of 363 patients were enrolled in the study; 173 patients with a mean age of $76.3 \pm 73.8$ months were imaged with standard dose protocols with FBP reconstruction, and 190 patients with a mean age $67.6 \pm 67.9$ months were imaged with low-dose protocols with IR reconstruction. Thirty-seven patients were excluded due to excessive motion, multiple implanted leads, or incorrect protocol. Age group and age were not significantly different (Table 1), and although sex difference did not achieve statistical significance, approximately two-thirds of the patients were male.

\section{Radiation Dose}

The use of IMR reconstructions allowed diagnostic images using lower dose parameters. The mean estimated $\mathrm{CTDI}_{\mathrm{vol}}$ was significantly reduced in the IMR group, $24.4 \pm 3.1 \mathrm{mGy}$ versus the FBP

Table 1: Patient demographics by age group, age, sex, and scanner ${ }^{\mathrm{a}}$

\begin{tabular}{lcccc}
\hline & IMR $(\boldsymbol{n}=190)$ & FBP $(\boldsymbol{n}=173)$ & Total $(\boldsymbol{n}=363)$ & $P$ Value \\
\hline Age group & & & & $.50^{\mathrm{b}}$ \\
$\quad$ Younger than 1.5 years & $65(34.2 \%)$ & $48(27.7 \%)$ & $113(31.1 \%)$ & \\
1.5-6 years & $60(31.6 \%)$ & $58(33.5 \%)$ & $118(32.5 \%)$ & \\
7-12 years & $24(12.6 \%)$ & $29(16.8 \%)$ & $53(14.6 \%)$ & \\
Older than 13 years & $41(21.6 \%)$ & $38(22.0 \%)$ & $79(21.8 \%)$ & \\
Age (mean) (SD) (months) & $67.6(67.9)$ & $76.3(73.8)$ & $71.8(70.8)$ & $.24^{\mathrm{c}}$ \\
Sex & & & & $.05^{\mathrm{b}}$ \\
$\quad$ Female & $81(42.6 \%)$ & $56(32.4 \%)$ & $137(37.7 \%)$ & \\
Male & $109(57.4 \%)$ & $117(67.6 \%)$ & $226(62.3 \%)$ & \\
\hline
\end{tabular}

${ }^{a}$ Data are number (percentage) unless otherwise indicated.

${ }^{b} \chi^{2}$ test.

${ }^{c}$ Equal variances $t$ test. group, $31.1 \pm 6.0 \mathrm{mGy}(P<.001)$. In addition, the $\mathrm{CTDI}_{\mathrm{vol}}$ was significantly reduced in each of the 4 individual age groups, $27 \%$, $25 \%, 13 \%$, and $12 \%$ (Table 2), with the largest dose savings observed in the younger-than-1.5 years and 1.5- to 6-year age groups.

\section{Reconstruction Times}

The average study reconstruction time for the FBP-group was 100.9 seconds (range, 64-237 seconds), and for the IMR group, it was 147.0 seconds (range, $125-473$ seconds) $(P<.001)$.

\section{Quantitative Analysis}

$\mathrm{SNR}_{\text {Thalamus }}$, $\mathrm{SNR}_{\text {White Matter }}$, and CNR were significantly improved in the IMR groups compared with the FBP groups approximately 2 -fold, with representative images and sample measurements in Fig 1 and group results summarized in Table 2.

\section{Qualitative Analysis}

A random number generator was used to select 5 patients from each of the age groups imaged on each scanner $(n=40)$. Subjective image quality, as it pertains to anatomic detail and graywhite matter differentiation, was scored, on average, $2.3 \pm 0.6$ (fair-to-average) for those standard-dose studies reconstructed with FBP, and $3.6 \pm 0.6$ (average-to-good) for those lower-dose studies reconstructed with IMR $(P<.001)$. Each physician independently scored those studies reconstructed with IMR as superior to those reconstructed with FBP (3.6 \pm 0.8 versus $1.9 \pm 0.8$ and $3.7 \pm 0.6$ versus $2.8 \pm 0.6(P<.001)$. However, score agreement between the individual physicians was low, with an intraclass correlation coefficient of 0.399 and a correlation coefficient $r=0.43$ for the FBP groups, and an intraclass correlation coefficient of 0.345 and $r=0.37$ for the IMR groups (Table 3).

\section{DISCUSSION}

In our patient population, we demonstrate statistically significant relative $\mathrm{CTDI}_{\mathrm{vol}}$ dose reduction in all 4 patient age groups undergoing emergency unenhanced head CT examinations, 1.5 years or younger, $1.5-6$ years, $7-12$ years, and 13 years and older, of $27 \%, 25 \%, 13 \%$, and $12 \%$ respectively, using low-dose protocols with IMR image reconstruction, compared with patients imaged using standard protocols and FBP image reconstruction, with statistically improved quantitative and qualitative image quality (Fig 1). The

Table 2: Mean (SD) dose estimates and objective image-quality measures by age group and scanner

\begin{tabular}{|c|c|c|c|c|c|c|c|c|}
\hline & \multicolumn{2}{|c|}{ Younger Than $1.5 \mathrm{yr}$} & \multicolumn{2}{|c|}{$1.5-6 \mathrm{yr}$} & \multicolumn{2}{|c|}{$7-12 \mathrm{yr}$} & \multicolumn{2}{|c|}{ Older Than $13 \mathrm{yr}$} \\
\hline & FBP & IMR & FBP & IMR & FBP & IMR & FBP & IMR \\
\hline$C T D I_{\text {vol }}$ & $29.9(5.5)$ & $21.8(0.1)$ & $30.6(5.5)$ & $22.8(0.0)$ & $31.5(6.2)$ & $27.5(0.0)^{a}$ & $33.3(6.9)$ & $29.2(0.6)^{\mathrm{a}}$ \\
\hline $\mathrm{SNR}_{\mathrm{THAL}}$ & $9.3(2.3)$ & $22.0(5.3)$ & $8.6(1.8)$ & $20.2(3.9)$ & $8.8(2.3)$ & $18.7(3.2)$ & $8.6(2.2)$ & $17.9(3.5)$ \\
\hline $\mathrm{SNR}_{\mathrm{WM}}$ & $8.3(2.3)$ & $16.5(3.8)$ & $8.2(2.3)$ & $15.8(3.3)$ & $7.8(1.3)$ & $16.9(4.3)$ & $7.9(2.4)$ & $14.9(2.9)$ \\
\hline CNR & $2.1(0.9)$ & $4.9(1.3)$ & $2.1(1.1)$ & $4.4(0.9)$ & $2.0(1.0)$ & $3.7(0.9)$ & $1.8(0.6)$ & $3.6(1.0)$ \\
\hline
\end{tabular}

Note:-THAL indicates thalamus; WM, white matter; vol, volume.

a $P$ value $<.003$. For all other results, $P$ value $<.001$, equal variances $t$ test. 
implication is that further dose reduction with even lower-dose techniques and maintained, rather than improved, image quality is possible with IMR.

The average reconstruction time with IMR was 46 seconds (46\%) longer than with standard FBP reconstruction, but with no practical effect on work flow. Willemink et $\mathrm{al}^{8}$ likewise reported a 35\% increased reconstruction time of hybrid iDose4 (Philips Healthcare) in abdominal CT examinations. Reconstruction times can be expressed in slices per second; however, the number of slices per second is not a linear function because there is a longer lag time until the first image. In our study, the FBP reconstructions averaged 5.2-5.6 slices/second, and the IMR reconstructions, 3.4-3.6 slices/second. As computer-processing speeds improve, image-reconstruction times with IMR should continue to decrease.

Multiple studies have reported success in reducing relative radiation doses in pediatric cohorts undergoing head CT examinations, ranging from $24 \%$ to $48 \%$, using low-dose protocols with hybrid IR image reconstruction versus standard-dose FBP protocols. ${ }^{9-15}$ Vorona et al, 2013, ${ }^{16}$ reported that $20 \%$ adaptive statistical iterative reconstruction (ASIR) could allow 22\% relative dose reduction in pediatric head CT without affecting image quality. Kilic et al, 2013, ${ }^{17}$ demonstrated a $29 \%$ dose reduction with lowdose 30\% ASIR versus standard-dose FBP in 305 pediatric head CT examinations with maintained diagnostic quality and image sharpness, but they noted decreased SNR and increased white
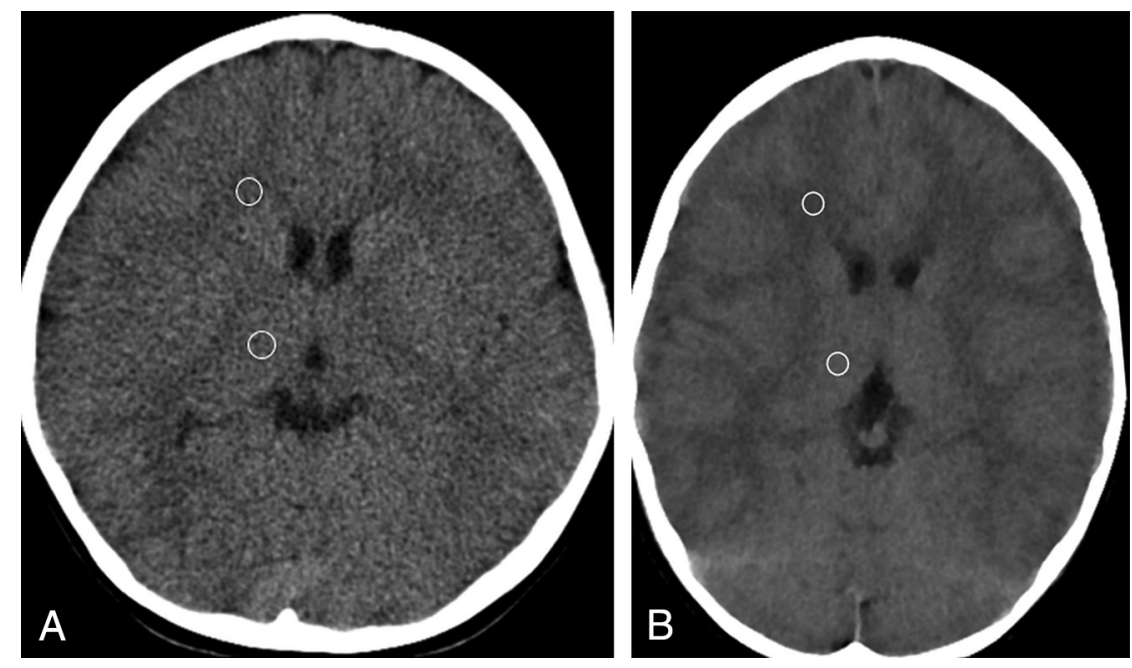

FIG 1. A, A 5-year-old girl bitten by a dog with scalp lacerations. Unenhanced head CT 3-mm axial image obtained using standard-dose protocol with FBP reconstruction. ROls were placed on the thalamus and frontal white matter: thalamus, $32.3 \pm 4.1 \mathrm{HU}$; SNR, 7.9; white matter, $26.8 \pm 3.9 \mathrm{HU}$; SNR, 6.9; CNR, 1.4; CTDI ${ }_{\text {vol }}, 28.7$ mGy. B, A 5-year-old boy who hit his head on concrete. Unenhanced head CT 3-mm axial image obtained using a low-dose age-based protocol with IMR reconstruction. ROls were placed on the thalamus and frontal white matter: thalamus, $31 \pm 1.5 \mathrm{HU}$; SNR, 20.7; white matter, $25 \pm 1.5 \mathrm{HU}$; SNR, 16.7; CNR, 4.1; CTDI ${ }_{\text {vol }}, 22.8 \mathrm{mGy}$. SNR and CNR improved 2-fold with IMR, with a $21 \%$ decrease in CTDI ${ }_{\text {vol }}$. matter noise in the IR images. McKnight et al, 2014, ${ }^{18}$ showed a relative dose reduction of $28 \%$ and $48 \%$ in pediatric head CT examinations in patients $3-12$ years of age and older than 12 years of age, respectively, using 30\% ASIR. Thomas et al, 2018, ${ }^{19}$ reported a relative dose reduction of $26 \%$ in pediatric head CT examinations using hybrid IR iDose4 strength level 2 reconstruction versus standard-dose FBP, with improved SNR and CNR. However, subjective image-quality reviewers preferred the FBP images for noise in the older-than-13-year group, and image sharpness in the 7- to 12-year and older-than-13-year groups. ${ }^{19}$ Kim et al, 2017, ${ }^{20}$ reported that the use of hybrid IR ASIR-V (GE Healthcare) allowed a $12.8 \%-34 \%$ dose reduction and lower noise and higher CNR in both supratentorial and posterior fossa structures in patients younger than 3 years of age and 3-15 years of age, with improved image sharpness on qualitative review. Ono et al, 2016, ${ }^{21}$ reported 78 children 5 years of age and younger undergoing emergent noncontrast head CT reconstructed with FBP and 2 strengths of hybrid sinogram-affirmed iterative reconstruction. SNR and CNR were highest in the IR groups, as were subjective image-quality scores for gray-white matter differentiation and artifacts from the skull.

Relatively few studies have assessed IMR in pediatric head CT examinations. To our knowledge, no prior study has compared pediatric head CT examinations reconstructed using IMR and FBP. Notohamiprodjo et al, 2015, ${ }^{22}$ reported improved subjective image quality, decreased artifacts, and significantly improved SNR and CNR in head CT examinations reconstructed with MBIR compared with hybrid IR (ASIR) and proposed the potential of further reduction of the radiation dose with MBIR. den Harder et al, 2015, ${ }^{23}$ reviewed hybrid and modelbased IR in pediatric CT examinations and demonstrated improved noise reduction with MBIR and described variations of image textures depending on IR strength and reconstruction kernels. ${ }^{23}$ Smith et al, 2014, ${ }^{24}$ compared reduced-dose MBIR and hybrid IR 30\% ASIR in pediatric body CT and reported decreased noise and improved spatial resolution with MBIR, but noted an altered texture and decreased sharpness of trabecular bone with MBIR. Cheng et al, $2018,{ }^{25}$ examined the image quality of head and neck CTA in pediatric patients scanned at a lowered radiation dose $(80$ $\mathrm{kV}$ ) using decreased IV contrast volume and IMR image reconstruction and

Table 3: Mean subjective image-quality scores (SD) and average combined scores with frequency of image-quality scores given for FBP and IMR images ${ }^{\mathrm{a}}$

\begin{tabular}{lccccccc}
\hline & Combined & DR1 & DR2 & DR1 (Frequency) & DR2 (Frequency) & $\boldsymbol{r}$ & ICC \\
\hline FBP & $2.3(0.6)$ & $1.9(0.8)$ & $2.8(0.6)$ & $6 / 11 / 2 / 1 / 0$ & $0 / 6 / 13 / 4 / 0$ & 0.43 & 0.399 \\
IMR & $3.6(0.6)$ & $3.6(0.8)$ & $3.7(0.6)$ & $0 / 1 / 10 / 6 / 3$ & $0 / 1 / 4 / 15 / 0$ & 0.37 & 0.345 \\
$P$ value & $<.001$ & $<.001$ & $<.001$ & & & \\
\hline
\end{tabular}

Note:- ICC indicates intraclass correlation coefficient; DR1, radiologist 1; DR2, radiologist 2.

${ }^{a}$ Combined scores are in column 2. Scores are 1 (poor) to 5 (excellent). 

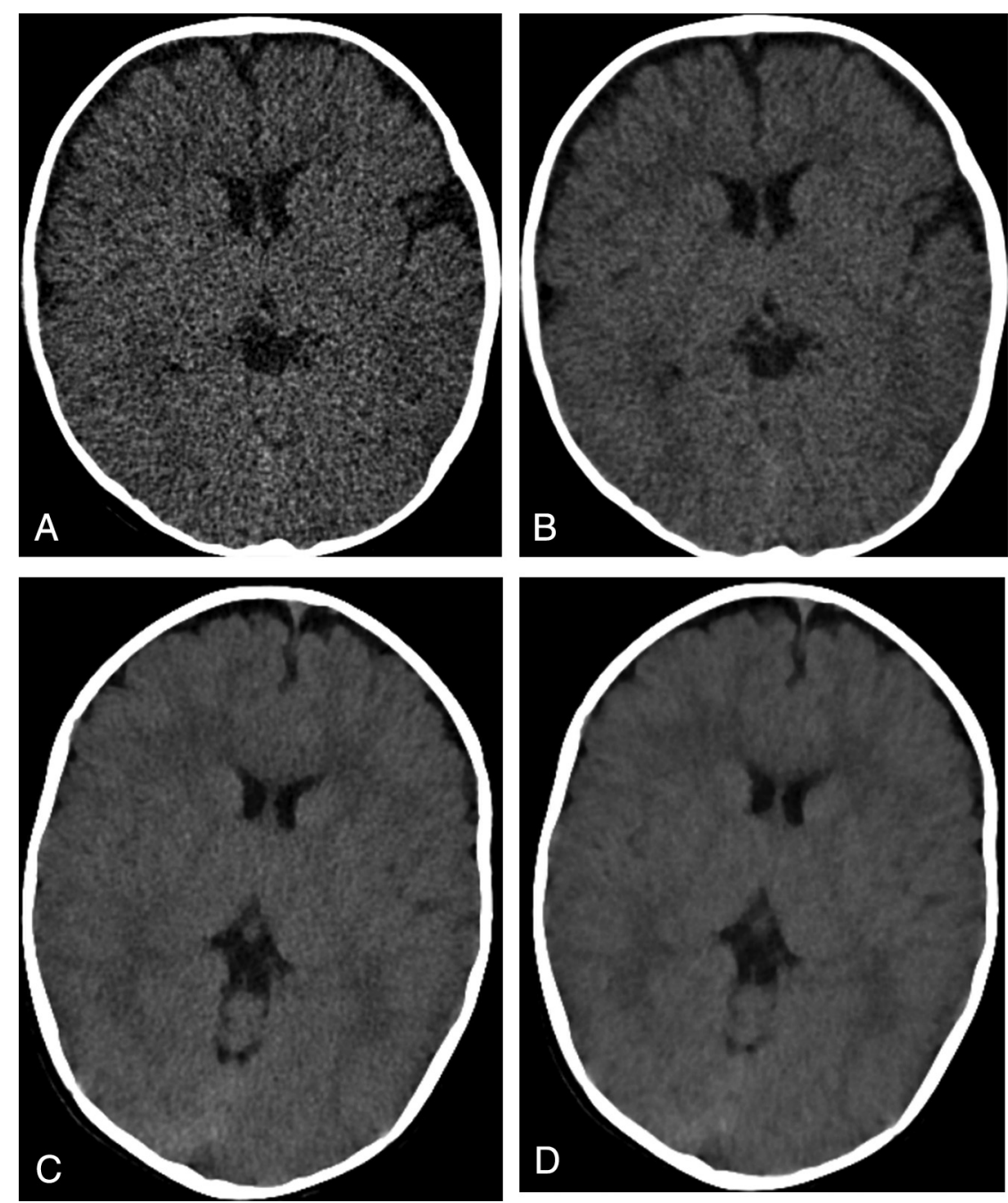

FIG 2. A 6-month-old female infant who fell from a couch. Axial noncontrast head CT using standard protocol and FBP reconstructed at 1-mm (top left) and 3-mm (top right) slice thickness. A, Thalamus, $29 \pm 7.5 \mathrm{HU}$; SNR, 3.9; WM, $26 \pm 7.5 \mathrm{HU}$; SNR, 3.5; CNR, 0.4; CTDI ${ }_{\text {vol }}, 25.5 \mathrm{mGy}$. B, Thalamus, $29 \pm 3.9 \mathrm{HU}$; SNR, 7.4; WM, $24 \pm 4.0 \mathrm{HU}$; SNR, 6.0; CNR, 1.3; CTDI ${ }_{v 0}, 25.5 \mathrm{mGy}$. The 1-mm FBP image has noticeably increased noise and a 69\% decrease in CNR compared with the $3-\mathrm{mm}$ FBP image, which lessens the diagnostic quality. A 6-month-old female infant with altered loss of consciousness and "not acting normally." Axial noncontrast images using low-dose protocol and IMR reconstructed at 1-mm (bottom left) and 3-mm (bottom right) slice thickness. C, Thalamus, $29 \pm 2.7 \mathrm{HU}$; SNR, 10.7; WM, $25 \pm 2.2 \mathrm{HU}$; SNR, 11.4; CNR, 1.6; CTDI ${ }_{\mathrm{vol}}, 21.8 \mathrm{mGy} . \mathrm{D}$, Thalamus, $29 \pm$

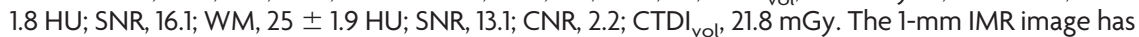
increased-but-acceptable noise, less than that in the 3-mm standard FBP image, and only a $27 \%$ decrease in CNR compared with the 3-mm IMR image. The 1-mm IMR has a more acceptable image texture and appears less waxy compared with the 3-mm IMR image.

found improved mean SNR and CNR, improved CT attenuation values of the arteries, and less noise compared with patients imaged at a higher $100 \mathrm{kV}$ with images reconstructed with FBP. Improved vessel attenuation, CNR, and reduced noise with IMR lend themselves to improved spatial resolution and 3D volumerendered models.

One common problem with model-based IR is altered image texture, which has been described as "waxy" or "glossy." To minimize this effect, our neuroradiologists rely on the 1-mm image set for primary diagnostic interpretation. The use of thinner image sets lessens the undesirable overly smooth image texture of the thicker images reconstructed using IMR, with relative maintained SNR, CNR, and acceptable noise (Fig 2).
A limitation of our study is that it was a retrospective review of patients, and we were unable to randomize patients and scanners. Our results demonstrated an average dose reduction of $22 \%$ with the use of IMR, which agrees with findings of other studies using hybrid IR. However, the concept of dose reduction is relative depending on reference protocols. Individual institutional results will vary depending on current protocols used, availability and type of IR used, physician noise tolerance, and imagetexture preferences.

There is an inherent limitation in assessing reconstruction times because the studies were performed on different scanners, and machine differences in hardware, processor clock speeds, and RAM could impact our observed reconstruction times.

Qualitative image-quality analysis may have been limited by the size of the random subsets evaluated. Lack of intraclass correlation coefficient agreement between our 2 reviewers may have been the result of how they were prepared to score image quality. Reviewers were only given a brief written and verbal guideline on how to rate the studies, but there was no hands-on prestudy training using case examples and no discussion on how to rate each parameter before conducting individual blinded reviews. Physician confidence and image-noise tolerance can be affected by experience, which we hoped to minimize by selecting radiologist reviewers, both with $\geq 16$ years' experience. Both reviewers individually scored the IMR images as superior to those reconstructed with FBP; however, 1 reviewer consistently scored comparable studies 1 full grade below that given by the second reviewer, also contributing to lack of an intraclass correlation coefficient. We used a 5-point rather that 4-point scale to score image quality, which added potential variability of possible scores. In addition, given the altered image texture of the IMR images, the reviewers could conceivably learn which reconstruction algorithm was used, possibly introducing bias.

Reported IMR subjective image-quality scores may have been underestimated because we used the 3-mm FBP and 3-mm IMR images for review and grading, which likely affected subjective image-quality scores, because our neuroradiology physicians routinely use the thin 1-mm IMR images for clinical interpretation. Liu et al, 2017, ${ }^{26}$ demonstrated that thin-slice 1-mm IMR images yielded the lowest noise, posterior fossa artifacts, and best CNR in 
unenhanced brain CT examinations, compared with 3-mm hybrid IR and 5-mm FBP reconstruction, and they had better sensitivity in the detection of small lacunar lesions. We did not examine posterior fossa image quality, which should be different from that in supratentorial measurements due to increased posterior fossa noise.

\section{CONCLUSIONS}

Study reconstruction times of urgent head CT examinations using IMR were statistically longer than those using FBP by $46 \%(P<$ .001 ), but without a practical effect on work flow. We demonstrated a relative dose reduction of $12 \%-27 \%$ using optimized low-dose head CT protocols with IMR, with the greatest dose savings seen in the youngest patient groups, with a 2 -fold improvement of SNR and CNR and statistically improved subjective image quality. Results suggest that further potential dose savings with maintained image quality are possible with IMR.

Disclosures: Richard N. Southard-UNRELATED: Consultancy: Philips Healthcare; Payment for Lectures Including Service on Speakers Bureaus: Philips Healthcare; OTHER RELATIONSHIPS: Koninklijke Philips NV: Master Enterprise Agreement between Philips Healthcare and Phoenix Children's Hospital. Dianna M.E. BardoUNRELATED: Consultancy: Koninklijke Philips NV, Comments: Master Enterprise Agreement*; Payment for Lectures Including Service on Speakers Bureaus: Koninklijke Philips NV, Comments: honoraria to my institution, travel support; Travel/ Accommodations/Meeting Expenses Unrelated to Activities Listed: Koninklijke Philips NV; Other: Thieme Publishing, Comments: Royalties. *Money paid to the institution.

\section{REFERENCES}

1. Wier LM, Hao Y, Owens PV, et al. Overview of Children in the Emergency Department, 2010. Statistical Brief\#157. June 2013. Healthcare Cost and Utilization Project (HCUP) Statistical Briefs [Internet]. Agency for Healthcare Research and Quality, Rockville, Maryland. https://www.ncbi.nlm.nih.gov/books/NBK154386/. Accessed March 26, 2019

2. Ryan M, Palasis S, Saigal G, et al. ACR Appropriateness Criteria head trauma: child. J Am Coll Radiol 2014;11:939-47 CrossRef Medline

3. Coakley F, Gould R, Yeh B, et al. CT radiation dose: what can you do right now in your practice? AJR Am J Roentgenol 2011;196:619-25 CrossRef Medline

4. Mahesh M. Advances in CT technology and application to pediatric imaging. Pediatr Radiol 2011;41(Suppl 2):493-97 CrossRef Medline

5. Smith A, Dillon W, Gould R, et al. Radiation dose-reduction strategies for neuroradiology CT protocols. AJNR Am J Neuroradiol 2007; 28:1628-32 CrossRef Medline

6. Seibert J. Iterative reconstruction: how it works, how to apply it. Pediatr Radiol 2014;44(Suppl 3):431-39 CrossRef Medline

7. Padole A, Khawaja DR, Kalra MK, et al. CT radiation dose and iterative reconstruction techniques. AJR Am J Roentgenol 2015;204: W384-92 CrossRef Medline

8. Willemink MJ, Schilham AM, Leiner T, et al. Iterative reconstruction does not substantially delay CT imaging in the emergency setting. Insights Imaging 2013;4:391-97 CrossRef Medline

9. Mirro AE, Brady SL, Kaufman RA. Full dose-reduction potential of statistical iterative reconstruction for head CT protocols in a predominantly pediatric population. AJNR Am J Neuroradiol 2016;37: 1199-205 CrossRef Medline

10. Harris M, Huckle J, Anthony D, et al. The acceptability of iterative reconstruction algorithms in head CT: an assessment of Sinogram Affirmed Iterative Reconstruction (SAFIRE) vs. filtered back projection (FBP) using phantoms. Journal of Medical Imaging and Radiation Sciences 2017;48:259-69 CrossRef

11. Ho C, Oberle R, Wu I, et al. Comparison of image quality in pediatric head computed tomography reconstructed using blended iterative reconstruction versus filtered back projection. Clin Imaging 2014;38:231-35 CrossRef Medline

12. Korn A, Fenchel M, Bender B, et al. Iterative reconstruction in head $\mathrm{CT}$ : image quality of routine and low-dose protocols in comparison with standard filtered back-projection. AJNR Am J Neuroradiol 2012;33:218-24 CrossRef Medline

13. Ren Q, Dewan SK, Li M, et al. Comparison of adaptive statistical iterative and filtered back projection reconstruction techniques in brain CT. Eur J Radiol 2012;81:2597-601 CrossRef Medline

14. Bodelle B, Klein E, Naguib NN, et al. Acute intracranial hemorrhage in CT: benefits of sinogram affirmed iterative reconstruction techniques. AJNR Am J Neuroradiol 2014;35:445-49 CrossRef Medline

15. Singh S, Kalra MK, Shenoy-Bhangle AS, et al. Radiation dose reduction with hybrid iterative reconstruction for pediatric CT. Radiology 2012;263:537-46 CrossRef Medline

16. Vorona G, Zuccoli G, Sutcavage T, et al. The use of adaptive statistical iterative reconstruction in pediatric head CT: a feasibility study. AJNR Am J Neuroradiol 2013;34:205-11 CrossRef Medline

17. Kilic K, Erbas G, Guryildirim M, et al. Quantitative and qualitative comparison of standard-dose and low-dose pediatric head computed tomography: a retrospective study assessing the effect of adaptive statistical iterative reconstruction. J Comput Assist Tomogr 2013;37:377-81 CrossRef Medline

18. McKnight $\mathrm{C}$, Watcharotone $\mathrm{K}$, Ibrahim $\mathrm{M}$, et al. Adaptive statistical iterative reconstruction: reducing dose while preserving image quality in the pediatric head CT examination. Pediatr Radiol 2014; 44:997-1003 CrossRef Medline

19. Thomas A, Southard R, Curran J, et al. Comparing fourth generation statistical iterative reconstruction technique to standard filtered back projection in pediatric head computed tomography examinations. J Comput Assist Tomogr 2018;42:475-81 CrossRef Medline

20. Kim HG, Lee HJ, Lee SK, et al. Head CT: image quality improvement with ASIR-V using a reduced radiation dose protocol for children. Eur Radiol 2017;27:3609-17 CrossRef Medline

21. Ono S, Niwa T, Yanagimachi N, et al. Improved image quality of helical computed tomography of the head in children by iterative reconstruction. J Neuroradiol 2016;43:31-36 CrossRef Medline

22. Notohamiprodjo S, Deak Z, Meurer F, et al. Image quality of iterative reconstruction in cranial CT imaging: comparison of model-based iterative reconstruction (MBIR) and adaptive statistical iterative reconstruction (ASiR). Eur Radiol 2015;25: 140-46 CrossRef Medline

23. den Harder AM, Willemink MJ, Budde RP, et al. Hybrid and modelbased iterative reconstruction techniques for pediatric CT. AJR Am J Roentgenol 2015;204:645-53 CrossRef Medline

24. Smith EA, Dillman JR, Goodsitt MM, et al. Model-based iterative reconstruction: effect on patient radiation dose and image quality in pediatric body CT. Radiology 2014;270:526-34 CrossRef Medline

25. Cheng B, Xing H, Lei D, et al. Impact of iterative model reconstruction combined with dose reduction on the image quality of head and neck CTA in children. Sci Rep 2018;8:12613 CrossRef Medline

26. Liu X, Chen L, Weiwei Q, et al. Thin-slice brain CT with iterative reconstruction algorithm for small lacunar lesions detection: image quality and diagnostic accuracy evaluation. Medicine (Baltimore) 2017;96:e9412 CrossRef Medline 